\title{
Molecular characterization and serodiagnostic potential of two serpin proteins in Psoroptes ovis var. cuniculi
}

\author{
Xiaobin Gu ${ }^{1 *}{ }^{*}$, Yuhang Chen ${ }^{1,2+}$, Chongyang Zhang ${ }^{1 \dagger}$, Yue Xie ${ }^{1}$, Nengxing Shen ${ }^{1}$, Ce Wang ${ }^{1}$, Xuan Zhou ${ }^{3}$, \\ Guangyou Yang ${ }^{1}$, Ran He ${ }^{1}$, Xuerong Peng ${ }^{4}$, Deying Yang ${ }^{3}$, Zhi He ${ }^{3}$ and Zhijun Zhong ${ }^{1}$
}

\begin{abstract}
Background: Psoroptes ovis var. cuniculi is a common ectoparasite of wild and domestic rabbits worldwide that causes economically devastating losses in commercial rabbit husbandry and significantly affects the overall health of rabbits. Serine proteinase inhibitor (serpin) is present in almost all organisms that are involved in host-pathogen interactions, inflammatory responses, and reproductive development, among others. However, very little research has been carried out on P. ovis var. cuniculi serpins.

Methods: Two serpin genes of P. ovis var. cuniculi (Pso c 27 and PsoSP2 CDNAs) were cloned and molecularly characterized. The transcriptional profiles and tissue localization of these two serpins in P. ovis var. cuniculi were investigated by quantitative real-time PCR and immunohistochemistry, respectively. The potential function of recombinant Pso C 27 and PsoSP2 (rPso c 27 and rPsoSP2) in the serodiagnosis of P. ovis var. cuniculi infestation in rabbits was evaluated using a newly devleoped indirect enzyme-linked immunosorbent assay.

Results: Both the 523-residue Pso c 27 and the 240-residue PsoSP2 proteins contained typical serpin domains and signatures. Both Pso c 27and PsoSP2 cDNAs were expressed throughout the life-cycle; specifically, the cDNAs showed significantly higher expression in female mites than in larva, nymph, and male mites (Pso c 27: $F_{(3,8)}=1935.953$, $P<0.0001$; PsoSP2: $\left.F_{(3,8)}=660.669, P<0.0001\right)$. The native Pso $c 27$ and PsoSP2 proteins localized in the ovary and mouthparts of adult female mites, respectively. Compared to rPsoSP2, rPso c 27 showed better diagnostic efficiency, with higher values of sensitivity, specificity, and area under the receiver operating characteristic curve (rPso c $27 \mathrm{vs}$ rPsoSP2: 96.0 vs 90.0\%; 90.91 vs 78.18\%; 0.988 vs 0.964, respectively). Moreover, rPso c 27 showed seropositivity in $80 \%$ of the rabbits as early as the 2 weeks post-infestation, prior to visible clinical signs and microscopy-positive of skin scrapings.
\end{abstract}

\footnotetext{
*Correspondence: guxiaobin198225@126.com

'Xiaobin Gu, Yuhang Chen and Chongyang Zhang contributed equally

to this work

${ }^{1}$ Department of Parasitology, College of Veterinary Medicine, Sichuan

Agricultural University, Chengdu 611130, Sichuan, People's Republic

of China

Full list of author information is available at the end of the article
}

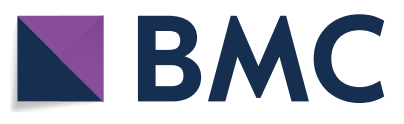

(C) The Author(s) 2020. This article is licensed under a Creative Commons Attribution 4.0 International License, which permits use, sharing, adaptation, distribution and reproduction in any medium or format, as long as you give appropriate credit to the original author(s) and the source, provide a link to the Creative Commons licence, and indicate if changes were made. The images or other third party material in this article are included in the article's Creative Commons licence, unless indicated otherwise in a credit line to the material. If material is not included in the article's Creative Commons licence and your intended use is not permitted by statutory regulation or exceeds the permitted use, you will need to obtain permission directly from the copyright holder. To view a copy of this licence, visit http://creativeco mmons.org/licenses/by/4.0/. The Creative Commons Public Domain Dedication waiver (http://creativecommons.org/publicdomain/ zero/1.0/) applies to the data made available in this article, unless otherwise stated in a credit line to the data. 
Conclusions: These results suggest that these two serpins may play essential roles in reproductive development, serum-feeding, and pathogenicity of P. ovis var. cuniculi. Compared to PsoSP2, Pso c 27 appears to be a potential anti-
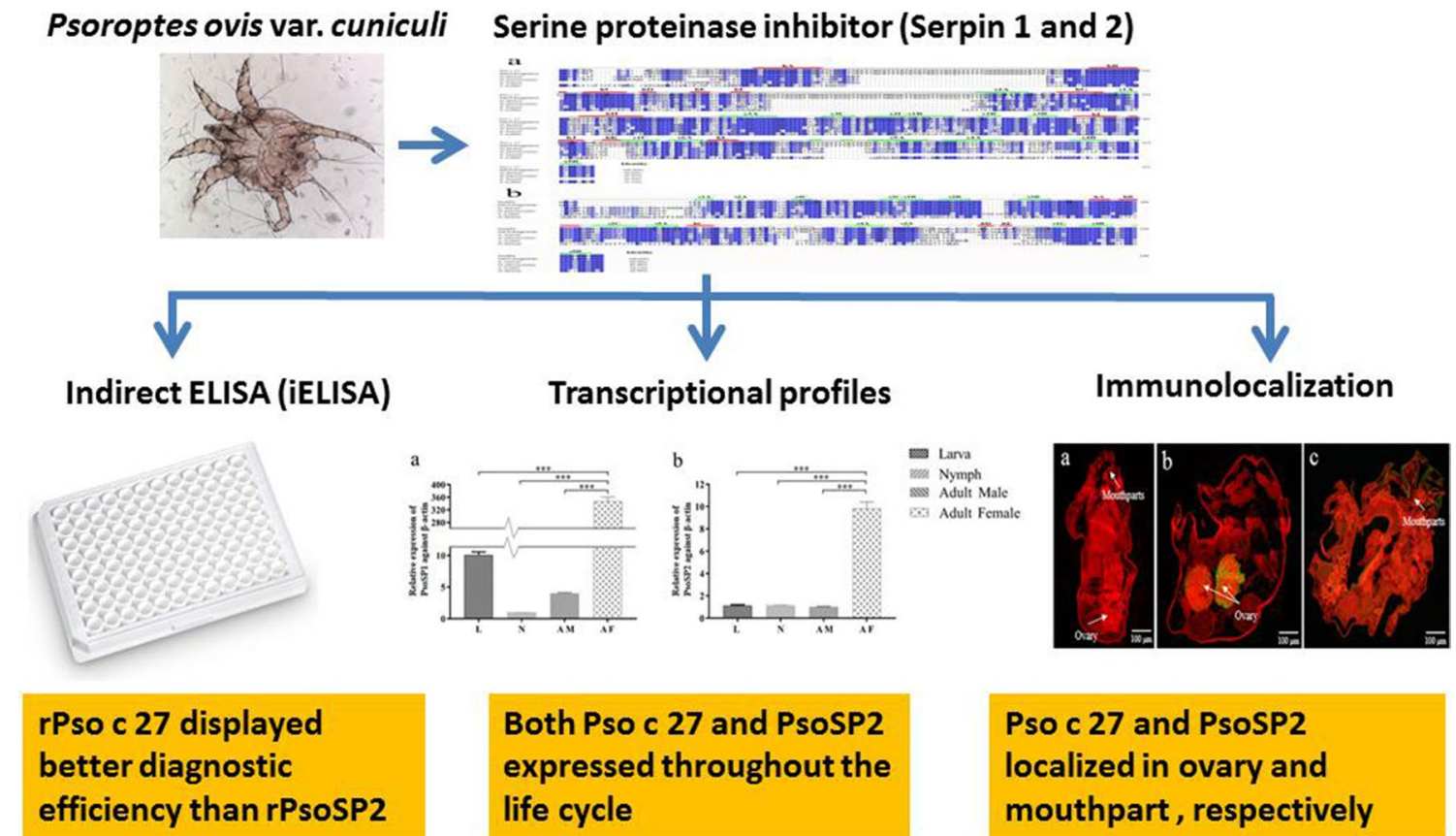

rPso c 27 displayed efficiency than rPsoSP2
Both Pso c 27 and PsoSP2 expressed throughout the life cycle
Pso c 27 and PsoSP2 localized in ovary and mouthpart, respectively

gen for serodiagnosis of P. ovis var. cuniculi infestation in rabbits, especially at the early stage of infestation.

Keywords: Psoroptes ovis var. cuniculi, Serpin, Transcriptional level, Tissue localization, Indirect ELISA, Serodiagnosis

\section{Background}

Psoroptes ovis var. cuniculi is a common ectoparasite of wild and domestic rabbits worldwide [1, 2]. This mite causes psoroptic mange in rabbits, which mainly presents as intense cutaneous inflammation, extreme pruritus, and crusted skin lesions [1, 2]. It signficantly affects the health welfare of rabbits and causes severe economic losses in commercial rabbit husbandry $[2,3]$.

The obligate ectoparasite $P$. ovis var. cuniculi is a non-burrowing mite that spends its entire life on the surface of host skin [4] where it feeds on serous fluids, lymph, and red blood cells [5]. The mite produces essential proteins to resist the host complement system to ensure successful feeding and self-proliferation. It also excretes allergens to promote the subsequent cutaneous inflammatory response [6,7]. Serine protease inhibitor (serpin) is expressed in almost all organisms. In arthropods, it has shown to possess a variety of fundamental physiological functions including anticoagulation, regulation of the inflammation response, and reproductive development, among others [8]. It also plays an essential role in the host-pathogen interaction [9]. Additionally, serpin may serve as a promising diagnostic antigen or vaccine candidate [10,11].
Recently, our analysis of transcriptomic data revealed that serpins exist in P. ovis var. cuniculi [12], but to our knowledge no further research on $P$. ovis var. cuniculi serpins has been reported to date. Analysis of these transcriptomic data led to the identication of two serpin genes of P. ovis var. cuniculi (Pso c 27 and PsoSP2 cDNAs) [12]. In the present study we focused on the function of these genes. We cloned and expressed the two recombinant Pso c 27 and PsoSP2 in prokaryotic expression vectors and performed sequence analysis. We also investigated the transcriptional profiles as well as tissue localization in mites, and their potential efficiencies in the diagnosis of $P$. ovis var. cuniculi infestation in rabbits were determined by indirect enzyme-linked immunosorbent assay (iELISA). This is a preliminary study aimed at elucidating the roles of these two proteins in P. ovis var. cuniculi and increasing our understanding of their functions.

\section{Methods}

Mite collection and RNA extraction

Psoroptes ovis var. cuniculi were harvested from an infested New Zealand White rabbit maintained at the Department of Parasitology, Sichuan Agricultural University (Sichuan, China). About 300 mites, consisting of 
pooled larvae, nymphs, and adults, were collected and processed for total RNA extraction.

\section{Expression and purification of two recombinant serpin proteins}

Total RNA was converted into cDNA using the PrimeScript RT reagent kit with gDNA Eraser (TaKaRa, Dalian, China). The two serpin genes were amplified from cDNA using the following primers: 5'-CGG GAT CCG CTC ATG TTG GTC AAC ATC-3' (forward) and 5'-C $\underline{\text { A }}$ AGC TTT TAA AAA TCA TGA ATT TCA CC- $3^{\prime}$ (reverse) for Pso c 27, where the underlining indicates restriction enzymes BamHI and HindIII; and 5'-CGG GAT CCT GAA TGC GAA TTC ATT GCT G-3' (forward) and 5'-CCC TCG AGT CAA AAT CCA TGC ATT TCA CC-3' (reverse) for PsoSP2, where the underlining indicates the restriction enzymes BamHI and XhoI. The cDNA fragments were sub-cloned into the pET32a $(+)$ plasmid (Invitrogen, Beijing, China). The recombinant proteins were expressed in Escherichia coli BL21 (DE3) and purified as described previously by $\mathrm{Gu}$ et al. [2]. The eluted fractions were concentrated using an Amicon Ultra Centrifugal Filter unit (Millipore, Billerica, MA, USA). Two purified serpin proteins were detected by $12 \%$ sodium dodecyl sulfate-polyacrylamide gel electrophoresis (SDS-PAGE).

\section{Sequence analysis}

The DNAMAN software package version 7.0 was applied to compare the similarity between paralogous genes, and the SignalP 5.0 tool (http://www.cbs.dtu.dk/Servi ces/SignalP/) was used to predict signal peptides. Transmembrane regions were analyzed using the Transmembrane Prediction Server (http://www.cbs.dtu.dk/servi ces/TMHMM-2.0). B-cell epitopes were predicted by BaCelLo (http://gpcr.biocomp.unibo.it/bacello/pred. $\mathrm{htm})$. The serine protease inhibition domains were analyzed using Inter-ProScan EMBL-EBI software (http:// pfam.xfam.org/). Amino acid sequences were aligned using MEGA5 [13]. Secondary structure predictions were performed by JPred 4.0 (http://www.compbio.dundee.ac. uk/jpred/). The neighbor-joining (NJ) tree, including values of 1000 replications resampled tests, was constructed by MEGA5 software [13].

\section{Rabbit sera}

Fifty $P$. ovis var. cuniculi-positive rabbit sera were collected from a farm located in Chengdu, Sichuan, China. Positivity for P. ovis var. cuniculi was confirmed in all rabbits based on the observation of ear scabs and micropscopic study of skin scrapings [14]. Twenty-five negative sera from $P$. ovis var. cuniculi-free rabbits were obtained from a farm without a history of psoroptic mange. For cross-reaction testing, an additional 30 sera samples, including Sarcoptes scabiei-positive sera, Eimeria spp.positive sera, and Cysticercus pisiformis-positive sera (ten samples of each) were provided by the Department of Parasitology, Sichuan Agricultural University.

\section{Preparation of polyclonal antibodies and western blotting} Polyclonal antibodies were obtained by experimental immunization with purified rPso c 27 and rPsoSP2, respectively. The products were raised following the procedures of $\mathrm{Gu}$ et al. [2], with slight modification. Briefly, rabbits were immunized with about $1 \mathrm{mg}$ purified recombinant protein four times by subcutaneous injection. Sera were collected from the marginal ear vein before immunization and 7 days after the fourth infection, and then purified by HiTrap Protein A affinity chromatography (Bio-scale $^{\mathrm{TM}}$ Mini UNOsphere SUPrA ${ }^{\mathrm{TM}}$ cartridge; BioRad, Hercules CA, USA) to obtain the immunoglobulin G (IgG) of anti-rPso c 27 and anti-rPsoSP2.

Two purified recombinant proteins were separated by $12 \%$ SDS-PAGE and transferred to the nitrocellulose membranes using Trans-Blot SD Semi-Dry Transfer Cell (Bio-Rad). The membranes were then blocked using 5\% skimmed milk powder for $2 \mathrm{~h}$, following which they were washed three times (each wash $5 \mathrm{~min}$ ) in TBST $(0.02 \mathrm{M}$ Tris- $\mathrm{HCl}, \mathrm{pH}$ 7.6, $0.15 \mathrm{M} \mathrm{NaCl}, 0.05 \%$ Tween-20) and then incubated with rabbit anti-P. ovis var. cuniculi antibody, anti-rPso c 27 IgG, or anti-rPsoSP2 IgG (1:150 v/v) overnight at $4{ }^{\circ} \mathrm{C}$. Non-infested rabbit serum was used as a negative control. After washing three times with TBST, membranes were incubated with horseradish peroxidase (HRP)-conjugated goat anti-rabbit antibody (1:1000 dilution; Boster Bio-Project Co. Dalian, China) for $1 \mathrm{~h}$ at room temperature. Following another three washes with TBST, the signal was detected using an Enhanced HRP-DAB Chromogenic Substrate Kit (Tiangen, Beijing, China).

\section{Immunolocalization of two serpin proteins in adult female P. ovis var. cuniculi}

The immunolocalization of two serpin proteins were performed as previously described [15]. Briefly, adult female mites were collected, embedded in paraffin blocks, and sliced into $5-\mu \mathrm{m}$ histological sections. The sections were then treated with $0.01 \mathrm{M}$ citrate buffer and incubated with purified rabbit anti-rPso c 27 IgG, anti-rPsoSP2 IgG, or pre-immune IgG $(1: 200 \mathrm{v} / \mathrm{v})$. After three washes with phosphate buffered saline (PBS), sections were incubated with fluorescein isothiocyanate-conjugated goat antirabbit IgG $(1: 200 \mathrm{v} / \mathrm{v})$ and visualized using a fluorescent microscope (BX53; Olympus Corp., Tokyo, Japan). 


\section{Transcriptional profiles of serpin at different life stages of $P$. ovis var. cuniculi}

Total RNA was extracted from larvae, nymph, and adult (male/female) mites, respectively, using a MiniBest Universal RNA Extraction kit (Takara Bio Inc., Shiga, Japan). Relative gene expression was evaluated by a two-step quantitative real-time PCR (qRT-PCR) using primers (i) Pso c 27: 5'-TGG CAG CAG TGG ATC AGA ATC ATC$3^{\prime}$ (forward) and 5'-AAT GCA ACA GCA ACA CTG TAT GGC-3' (reverse); PsoSP2: $5^{\prime}$-TCC TAC ATA CAC GTC CAT CAA CA- $3^{\prime}$ (forward) and $5^{\prime}$-TGG TAC AAT AGC GAC GGC TG- $3^{\prime}$ (reverse). The $\beta$-actin gene was used as a housekeeping control to correct for the relative fluorescence signal of the target genes, using primers $5^{\prime}$-TGA ATT GCC TGA TGG TCA AG-3' (forward) and $5^{\prime}$-TGG CGA ACA AGT CTT TAC GG-3' (reverse). Gene transcription was assessed according to the manufacturers' recommendations of the real-time PCR system (LightCycler ${ }^{\circledR} 96$ System; Roche Group, Basel, Switzerland) and the SYBR Premix Ex Taq II kit (TaKaRa). Each sample was performed in triplicate. An equal volume of $\mathrm{ddH}_{2} \mathrm{O}$ replaced the template cDNA as a blank control. Thermal cycling was performed as follows: one cycle at $95{ }^{\circ} \mathrm{C}$ for $30 \mathrm{~s}, 95{ }^{\circ} \mathrm{C}$ for $5 \mathrm{~s}, 58{ }^{\circ} \mathrm{C}$ for $30 \mathrm{~s}$; followed by 40 cycles at $95{ }^{\circ} \mathrm{C}$ for $5 \mathrm{~s}, 59{ }^{\circ} \mathrm{C}$ for $15 \mathrm{~s}$, and $95{ }^{\circ} \mathrm{C}$ for $1 \mathrm{~s}$. Melting curves were plotted, and relative expression levels of the target genes were calculated by the $2^{-\Delta \Delta \mathrm{Ct}}$ method.

\section{Establishment of an indirect ELISA (iELISA)}

The establishment of iELISA was performed as described by Crowther [16]. The concentrations of antigen and primary serum samples were determined by the checkerboard titration tests. Briefly, the purified proteins were diluted twofold in $0.1 \mathrm{M}$ carbonate buffer $(\mathrm{pH} 9.6)$ to the different concentrations and placed in 96-well plates at $100 \mu \mathrm{l} /$ well overnight at $4{ }^{\circ} \mathrm{C}$. The plates were washed three times in PBS containing Tween-20 (PBST; $\mathrm{pH} 7.4$ ) (5 min per wash), then incubated first with $5 \%$ (w/v) skimmed milk powder at $37{ }^{\circ} \mathrm{C}$ for $90 \mathrm{~min}$, then with $100 \mu \mathrm{l}$ of the two-fold gradient dilution of $P$. ovis var. cuniculi-positive and -negative serum samples (dilution ranging from $1: 20$ to $1: 320$ ) at $37^{\circ} \mathrm{C}$ for $1 \mathrm{~h}$. The plates were subsequently washed 3 times and incubated $1 \mathrm{~h}$ at $37^{\circ} \mathrm{C}$ with $100 \mu \mathrm{l} \mathrm{HRP-labeled} \mathrm{goat} \mathrm{anti-rabbit}$ IgG (1:3000 dilution with 0.01 M PBS) (Boster Bio-Project Co.). After 4 washes, $100 \mu \mathrm{l}$ of TMB chromogenic solution (Tiangen, Beijing, China) was added at $37{ }^{\circ} \mathrm{C}$ for $20 \mathrm{~min}$, then the reaction was stopped with $100 \mu \mathrm{l} /$ well of $2 \mathrm{M} \mathrm{H}_{2} \mathrm{SO}_{4}$. Optical densities (OD) were read at $450 \mathrm{~nm}$ by a microplate reader (Thermo Fisher Scientific, Waltham, MA, USA). The optimal working conditions were determined based on the highest positive : negative serum value. The cut-off value of iELISA was determined as the mean $\mathrm{OD}_{450}$ value plus three standard deviations (SD) using 25 negative serum samples from naïve rabbits [2].

To further evaluate the feasibility of the iELISA, $50 P$. ovis var. cuniculi-positive serum samples were assessed by the iELISA, and the sensitivity was calculated as (ELISA-positive samples $\times 100$ )/true $P$. ovis var. cuniculipositive serum samples [2]. Thirty serum samples from rabbits infected with S. scabiei, Eimeria spp., and C. pisiformis, respectively (10 samples for each species) were used to evaluate the cross-activity of the iELISA. Twentyfive $P$. ovis var. cuniculi-negative serum samples from naïve rabbits and 30 serum samples in the cross-activity assay were used to determine the specificity of the iELISA, which was calculated as (ELISA-negative samples $\times 100$ )/true $P$. ovis var. cuniculi-negative serum samples [2]. The area under the receiver operating characteristic curve (AUC), a graph of the sensitivity (true positive rate) versus 1-specificity (false positive rate), was the calculated using MedCalc 19.0.7 [17].

The repeatability (intra-assay variability) and reproducibility (inter-assay variability) of the iELISA were evaluated using three $P$. ovis var. cuniculi-positive serum samples, substantially as previously described [18].

\section{The experimental infestation of rabbits with $P$. ovis var. cuniculi and serological testing using the newly developed iELISA}

Rabbits infected with $P$. ovis var. cuniculi were treated strictly as previously described [2]. Briefly, ten 3-monthold naive New Zealand rabbits (5 females, 5 males) were infested with $P$. ovis var. cuniculi, and three non-infested rabbits were used as controls. Serum samples from 13 rabbits were collected at weeks $0,1,2,3$, and 4 . Afterwards, a total of 65 serum samples (50 from the $P$. ovis var. cuniculi-infestated rabbits and 15 from the noninfested rabbits) were examined by the newly developed optimal iELISA method. Each serum sample was tested in triplicate and analyzed in one ELISA plate, with positive and negative controls also contained in the plate.

\section{Statistical analysis}

All data are presented as the mean \pm SD. And statistical differences between groups were evaluated using MannWhitney U-tests in SPSS software v.17.0. (IBM Corp. Armonk, NY, USA). $P$ values of $<0.05$ were considered to be statistically significant.

\section{Results}

Sequence analyses of the two serpins

The 1572-bp open reading frame (ORF) in Pso c 27 cDNA (GenBank: MT707535) encodes 523 amino acids 
(aa), and the 723-bp ORF in PsoSP2 cDNA (GenBank: MT707536) encodes 240 aa. The Pso c 27 protein contains a signal peptide but no transmembrane region, whereas PsoSP2 appears to contain a transmembrane region but no signal peptide.
Pso c 27 and PsoSP2 shared 31.33-50.85 and 28.99$69.92 \%$ aa sequence identity with orthologs in other mites (Fig. 1). Interestingly, Pso c 27 and PsoSP2 shared $100 \%$ aa sequence identity with the reported serpin-like proteins of $P$. ovis PSOVI22g04610 and PSOVI22g04560, respectively [19] (Fig. 1). Pso c 27 consisted of 12 helices

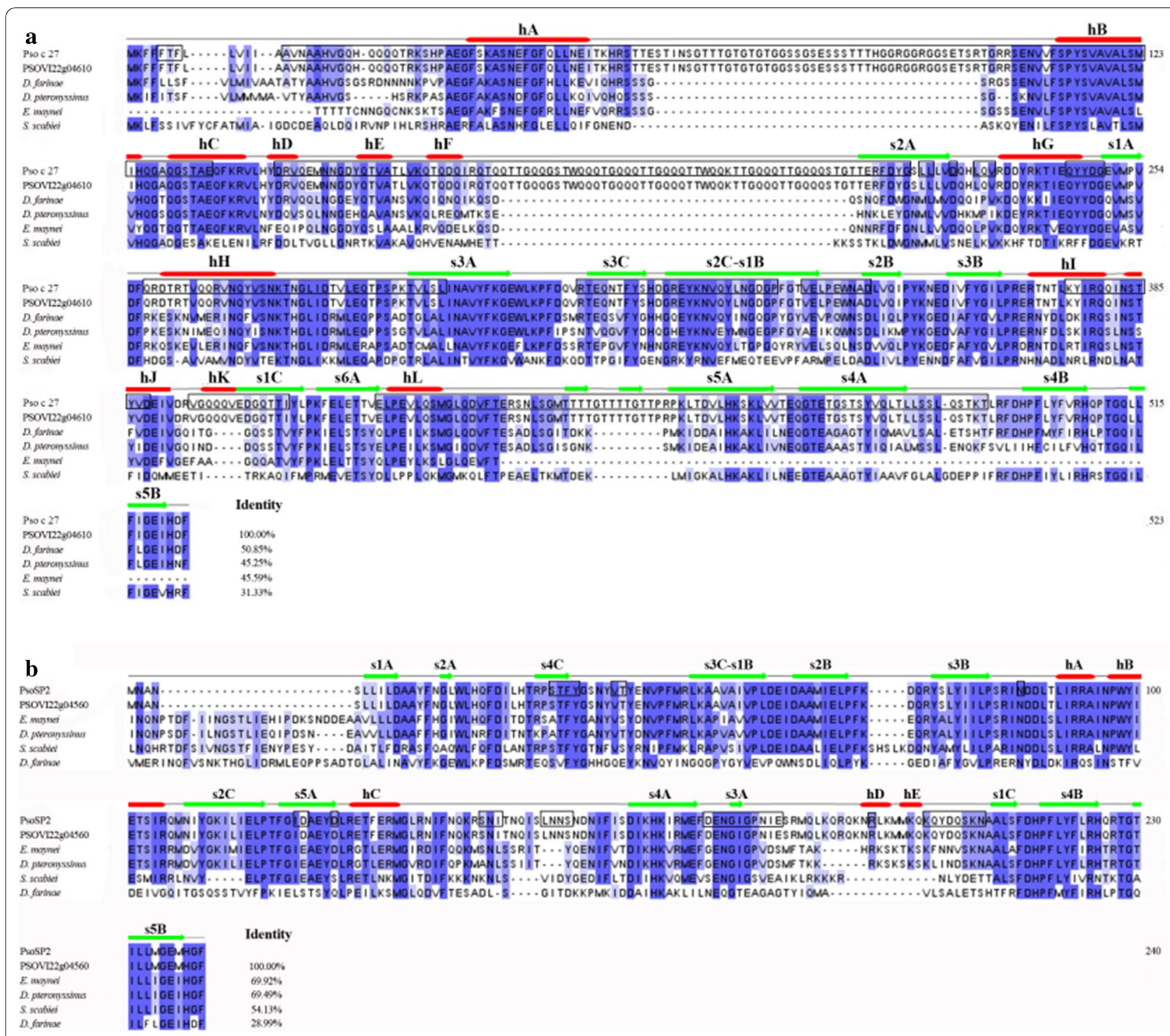

Fig. 1 Multiple sequence alignment of Psoroptes ovis var. cuniculi serpin genes Pso c 27 (a) and PsoSP2 (b). a Pso c 27. Multiple sequence alignment of the deduced amino acid sequence of Pso c 27 with homologous sequences of related proteins of other parasites: P. ovis (PSOVI22g04610), Dermatophagoides farinae (GenBank: AlO08851.1), D. pteronyssinus (GenBank: ATI08940.1), Euroglyphus maynei (GenBank: OTF72764.1), and Sarcoptes scabiei (GenBank: AEB40052.1). b PsoSP2. Multiple sequence alignment of the deduced amino acid sequence of PsoSP2 with homologous sequences of related proteins of other parasites: P. ovis (PSOVI22g04560), Euroglyphus maynei (GenBank: OTF74296.1), Dermatophagoides pteronyssinus (GenBank: XP_027200949.1), Sarcoptes scabiei (GenBank: KPM10873.1), and Dermatophagoides farinae (GenBank: AAP35082.1). Psoroptes ovis (PSOVI22g04610) and P. ovis (PSOVI22g04560) were obtained from the Online Resource for Community Annotation of Eukaryotes (OrcAE) (https://bioinformatics.psb.ugent.be/orcae/overview/Psovi). Helices are indicated with red horizontal bars. Horizonatal dark-green arrows along the sequence indicate sheets. Elements of secondary structure are labeled as: $h A$, $h B$, etc. A-helix, B-helix, etc., s1A, s2A, etc. strand 1 of the $A \beta$-sheet, strand 2 of the $A \beta$-sheet, etc. Consistent residues are highlighted with a dark-blue background, and consistent partial residues are highlighted with a light-blue background. B-cell epitopes are marked with a black box 
and three sheets, while PsoSP2 consisted of five helices and three sheets (Fig. 1a). A serpin domain was identified in the aa sequence from Arg78 to His493 for Pso c 27 and from Asn4 to Met237 for PsoSP2 [20]. Moreover, both proteins appeared to possess the specific serpin signature at the deduced aa sequences from 496 to 506 (LRFDHPFLYFV) for Pso c 27 and from 213 to 223 (LSFDHPFLYFL) for PsoSP2, respectively (Fig. 1a, b). The NJ tree revealed that Pso c 27 had the closest relationship with $P$. ovis-leukocyte elastase inhibitor-like protein (PSOVI22g04610), then clustered with D. farinae-Der f 27 allergen, $D$. pteroyssinus-Der f 27 -like allergen, and E. maynei-serpin (bootstrap frequency $[\mathrm{Bf}]=100 \%$ ), whereas PsoSP2 had the closest relationship with $P$. ovis serpin B5 (PSOVI22g04560) $(\mathrm{Bf}=100 \%)$, then grouped with E. maynei-serpin-like and D. pteroyssinus-serpin B10-like (Bf=98\%; Fig. 2).

\section{Expression and identification of two recombinant serpins} rPso c 27 were mainly present in the supernatant, with an expected size of $\sim 75 \mathrm{kDa}$, whereas rPsoSP2 principally present in insoluble inclusion bodies, with an expected size of $\sim 46 \mathrm{kDa}$ (including $\sim 18 \mathrm{kDa}$ His-tag fusion peptide from pET-32a) (Fig. 3). Western blotting showed that rPso c 27 and rPsoSP2 reacted with both $P$. ovis var. cuniculi-positive sera and the correspondent anti-serum IgG from rabbits, but not with negative sera, revealing a favourable reactivity and antigenicity (Fig. 3).
Tissue localization of two serpins in adult female $P$. ovis var. cuniculi

Native Pso c 27 and PsoSP2 were located in the ovary and mouthparts of female mites, respectively (Fig. 4b, c). No fluorescence signal was observed in adult female mites using pre-immunized rabbit IgG antibodies (Fig. 4a).

\section{Transcriptional profiles of the two serpins in P. ovis var. cuniculi}

The qRT-PCR data revealed that Pso c 27 and PsoSP2 cDNAs were expressed throughout the life-cycle of mites but that there was significantly higher expression in female mites than in larvae, nymphs, and male mites, respectively (Pso c 27, $F_{(3,8)}=1935.953, P<0.0001$; PsoSP2, $F_{(3,8)}=660.669, P<0.0001$ ) (Fig. 5).

\section{Serodiagnosis potential of two recombinant serpin proteins}

Using checkerboard titration, we determined that the optimal working conditions of the iELISA were $46.0 \mu \mathrm{g} /$ $\mathrm{ml}$ of rPso c 27, $64.5 \mu \mathrm{g} / \mathrm{ml}$ of rPsoSP2 for coated antigens, and a 1:100 dilution for rabbit sera. The cut-off values of $\mathrm{OD}_{450}$ were 0.633 for rPso c 27 and 0.490 for rPsoSP2.

The sensitivities were determined as the results of P. ovis var. cuniculi-positive sera, with $96.0 \%$ sensitivity for rPso c 27 (48/50) (Fig. 6a) and 90.0\% for rPsoSP2 (45/50) (Fig. 6b). The specificities were $90.91 \%$ for rPso c $27(50 / 55)$ and $78.18 \%$ for rPsoSP2 (43/55). Consequently, the AUC was 0.988 for rPso c 27-iELISA (95\% confidence interval [CI] CI 0.944-0.999) and 0.964 for

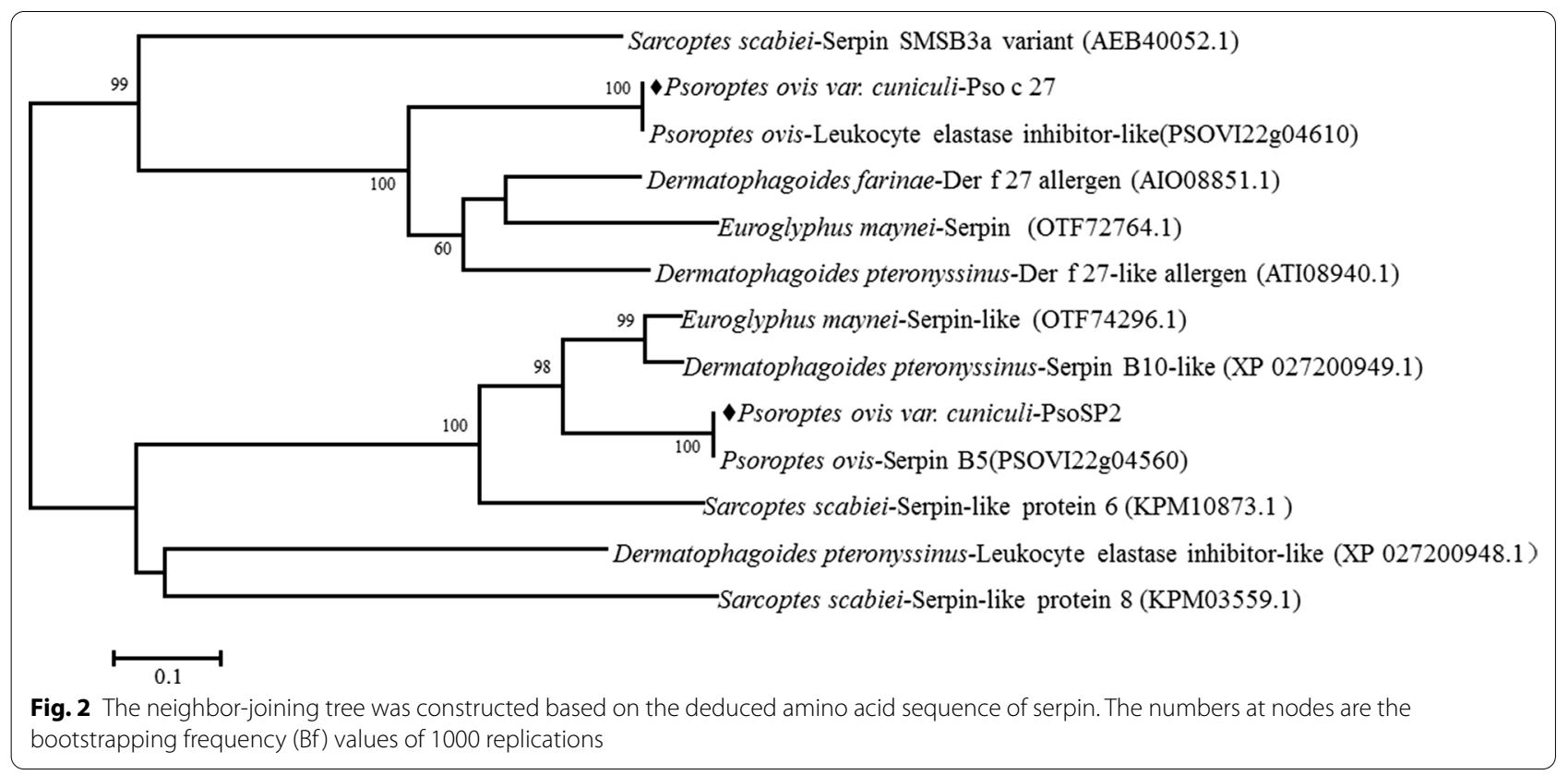




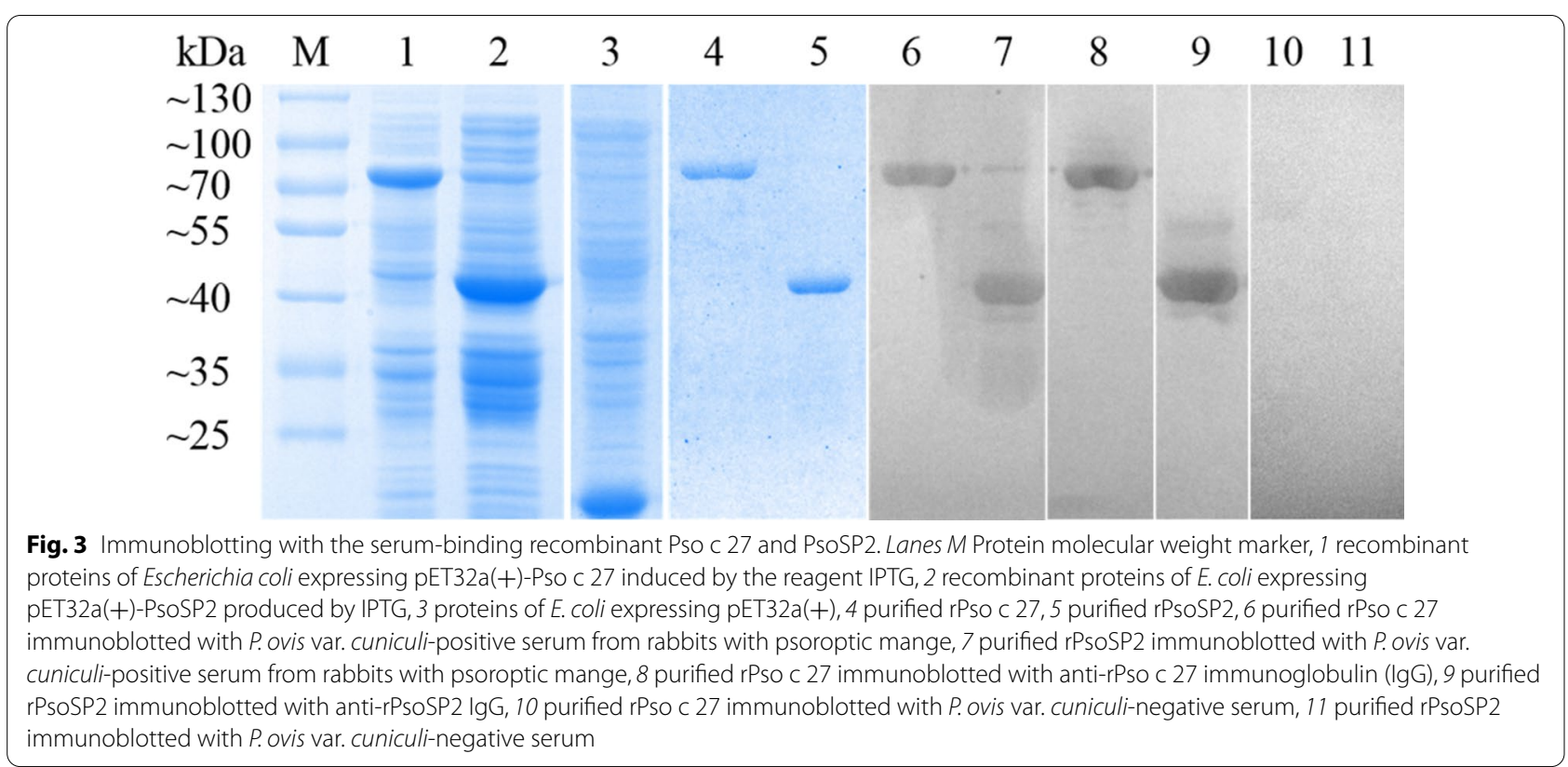
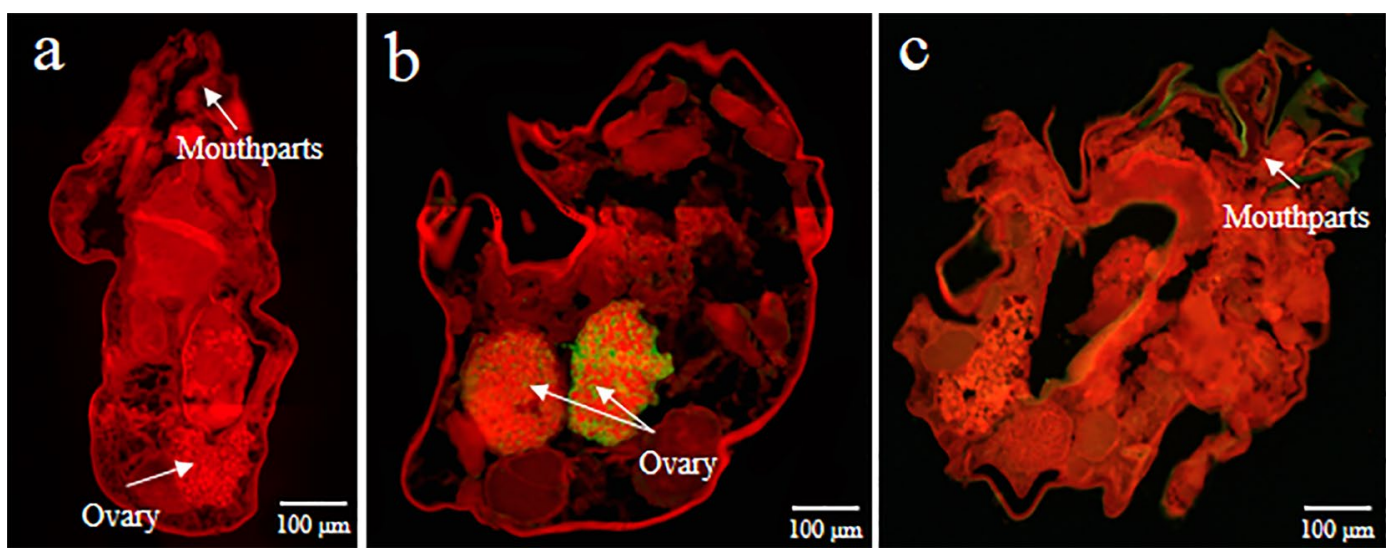

Fig. 4 Immunolocalization of Pso c 27 and PsoSP2 in the adult female of P. ovis var. cuniculi. a Incubation with the negative lgG of the rabbit before immunization, $\mathbf{b}$ incubation with the specific $\operatorname{lgG}$ of anti-rPso $\mathbf{2} 27, \mathbf{c}$ incubated with the specific $\lg \mathrm{G}$ of anti-rPsoSP2. All images were taken under a fluorescent microscope (magnification 100x)

rPsoSP2-iELISA (95\% CI 0.908-0.991), indicating that the rPso c 27-iELISA showed a better accuracy to detect specific antibodies against $P$. ovis var. cuniculi than the rPsoSP2-iELISA (Fig. 7). The intra- and inter-assay variabilities of rPso c 27 -iELISA were $<5 \%$, indicating that the newly developed rPso c 27-iELISA was stable and reproducible.

\section{Serodiagnostic test of rabbits experimentally infested with $P$. ovis var. cuniculi}

At 4 weeks post-infestation (p.i.), all infested rabbits were observed to have ear scabs. Skin scrapings also tested positive for P. ovis var. cuniculi. By rPso c 27-iELISA, the mean value of the anti-rPso $\mathrm{c} 27$ level from the infestation group revealed an increase from 1 to 4 weeks p.i. (Fig. 8). In the infestion group, anti-rPso c 27 positivity above the cut-off value was first detected in two of ten serum samples at 1 week p.i., following which the positivity gradually increased to $80 \%$ of samples $(8 / 10)$ at 2 and 3 weeks p.i., then up to $100 \%$ of samples $(10 / 10)$ at 4 weeks p.i. (Fig. 8). In the non-infestation group, the rate of anti-rPso c 27 antibody positivity remained below the cut-off value throughout the experiments. 

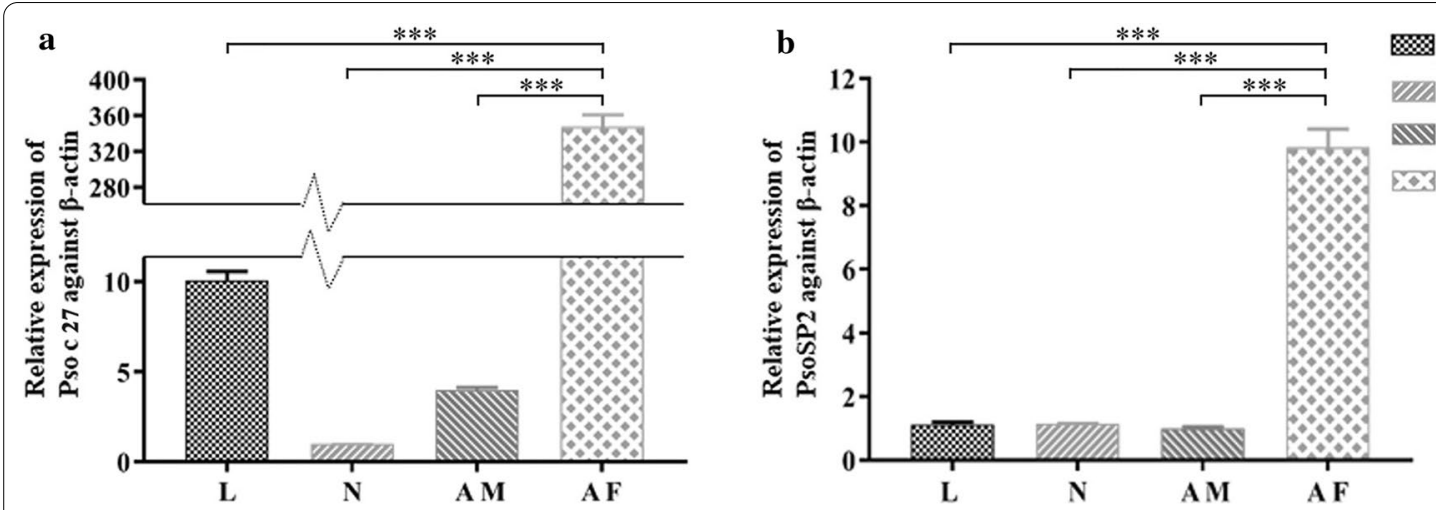

Larva

Nymph

Adult Male

Adult Female

Fig. 5 Relative transcriptional profiles of Pso c 27 (a) and PsoSP2 (b). The internal reference gene was $\beta$-actin. Data are represented as the mean with standard deviation (SD) from triplicate measures. L Larva, N nymph, AM adult male, AF adult female. Asterisks (***) indicate a statistically significant difference at $P<0.0001$
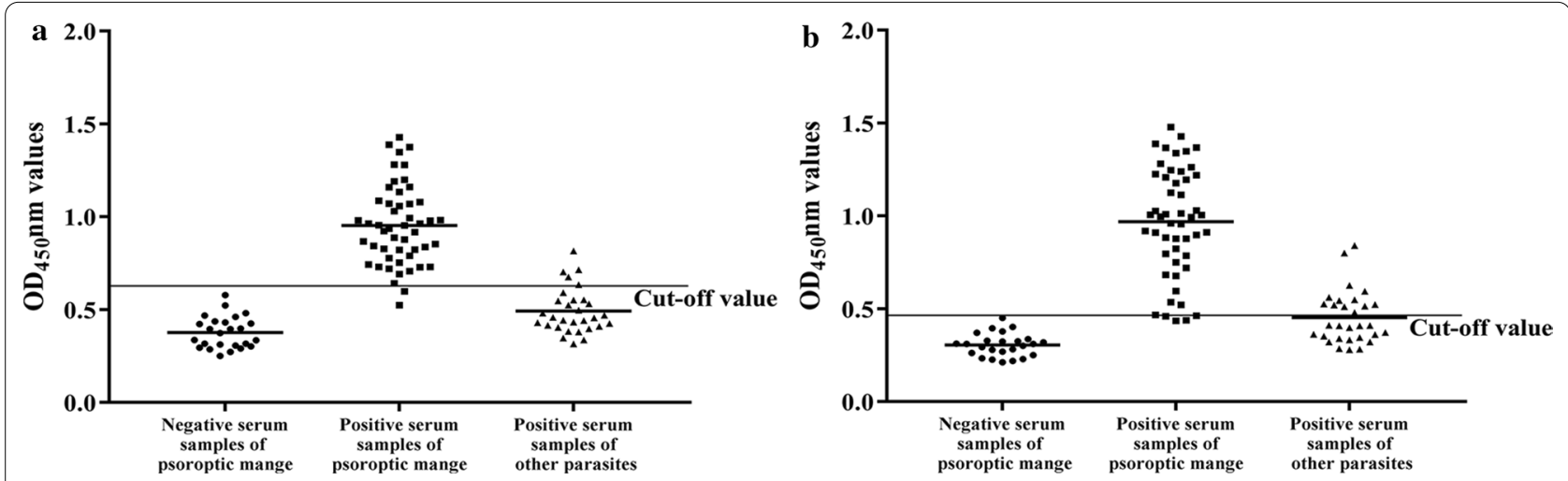

Fig. 6 Specificity, sensitivity,and cross-reactivity of rPso c 27 (a) and rPsoSP2 (b) by the newly developed indirect enzyme-linked immunosorbent assay (iELISA). The thin horizontal line represents the cut-off value (rPso c 27-iEISLA: 0.633; rPsoSP2-iELISA: 0.490). Statistically significant differences were compared between P. ovis var. cuniculi-positive serum and the other serum samples, including Eimeria spp.-positive, C. pisiformis-positive, $S$. scabiei-positive and P. ovis var. cuniculi-negative serum samples. OD Optical density. Asterisks $\left(^{* * *}\right)$ indicate a statistically significant difference at $P<0.0001$

\section{Discussion}

In the present study, two P. ovis var. cuniculi serpins were characterized, and the potential of their recombinant proteins was evaluated for serodiagnosis of $P$. ovis var. cuniculi infestation in rabbits. The Psoroptes mites used in the present study and our previous transcriptomic study are the same species, and the present findings confirmed the transcriptomic results of the earlier study in showing that two cloned serpin ORFs had 100\% identity with the assembled serpin sequences in the transcriptome data [12]. The predicted aa sequence showed the low overall identity of serpins compared those in other mites; however, these two target proteins were identified as typical serpins due to the presence of such features as the serpin domain and serpin signature in the $\mathrm{C}$-terminal end [20]. Pso c 27 shared $50.85 \%$ aa sequence identity with the newly characterized $D$. farinae Der f 27 allergen, which has been proven to orchestrate the pulmonary inflammatory response and mediate a Th2-type response in mice [21]. In addition, NJ analysis revealed that Pso c 27 is closely related to Der f 27. Based on the homology results and the genetic relationship between Pso c 27 and Der f 27, Pso c 27 may be considered to be an allergen of $P$. ovis var. cuniculi that is possibly associated with the instigation of the host cutaneous pro-inflammatory response [22]. This cutaneous inflammation has been shown to result in serum extravasation, thereby providing sufficient food for mite population growth, and to aggravate scabby lesions $[5,14]$. The expression of Pso c 27 and PsoSP2 in all stages of mites indicated that Pso c 27 and PsoSP2 possibly play an essential role in the development of $P$. ovis var. cuniculi. However, significant differences 


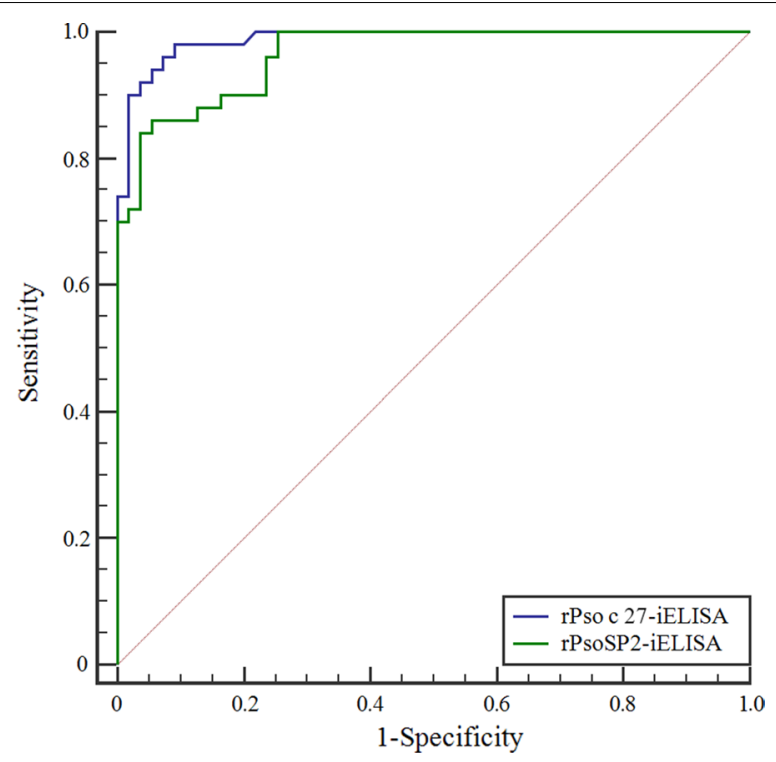

Fig. 7 The receiver operating characteristic (ROC) curves of the rPso c 27-iELISA and rPsOSP2-iELISA for the detection of antibodies against P. ovis var. cuniculi. The ordinate represents the sensitivity of the IELISA. The abscissa represents the 1-specificity of the iELISA. The purple line shows the mean area under the curve (AUC) plot of rPso c 27-iELISA, and the green line shows the mean AUC plot of rPsoSP2-iELISA

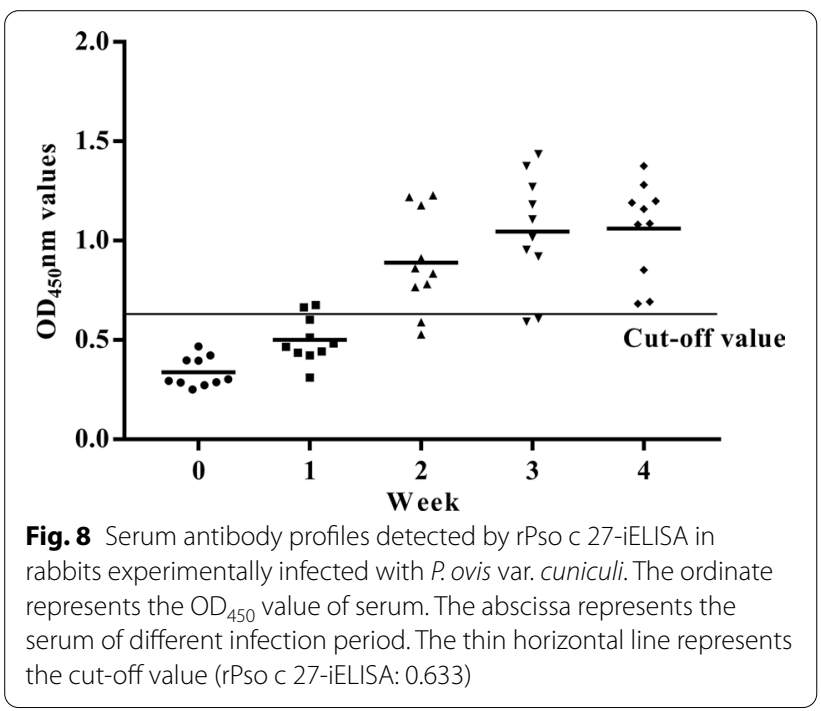

were seen for the transcription of Pso c 27 in the different life-cycle stages, and female mites showed the highest level of expression, with 347-fold change. In addition, the native protein was located in the ovary of female mites, indicating that Pso c 27 is possibly an essential factor in vitellogenesis $[19,23]$. This role of serpin in vitellogenesis has been proven in a recent study, which indicated that RNA interference (RNAi) of the serpin gene resulted in a reduction of yolk granule accumulation in Rhipicephalus haemaphysaloides [24].

Psoroptes mites are serum-feeding ectoparasites [5] and possess the ability to counter the host's complement attack. In this study, PsoSP2 showed homology to the $S$. scabiei serpin family genes $(20.98-54.13 \%$ aa sequence identity), some of which have been confirmed to inhibit the activation of complement pathways $[25,26]$. Moreover, the native PsoSP2 localized in the mouthparts of female mites and its cDNA expression throughout the life stages of mites suggest that PsoSP2 may appear to be vital in mites for anti-complement activity to successful serum-feeding $[5,9]$, and PsoSP2 could be a potential vaccine candidate.

Psoroptic mange spreads rapidly under crowded conditions and causes major morbidity in commercial rabbit husbandry in China [27]. Thus, timely diagnosis and treatment of $P$. ovis var. cuniculi infestation in rabbits are of paramount importance to reduce the risk of disease transmission and improve profitability. In China, the current diagnostic method for this disease, by examination of skin scrapings under the microscope, is extremely time-consuming and inefficient, especially for low densities of mites and sub-clinical infestations in rabbits. Thus, it is imperative to develop effective immunoreactive antigens for the rapid and accurate diagnosis of $P$. ovis var. cuniculi infestation in rabbits. Rabbits infested with $P$. ovis var. cuniculi can evoke a sero-specific antibody [1, 28], and this sero-specific antibody has been induced at the early phase of parasite infestation when rabbits appeared asymptomatic [1, 2]. Thus, the ELISA can be considered as an accurate method for detecting carriers of low densities of mites and/or sub-clinical infestations when compared with the microscopy of skin scrapings. In a previous study, serpin of Schistosoma mansoni was considered to be a promising species-specific diagnostic antigen in human schistosomiasis [10]. Therefore, in this study, we evaluated the serodiagnostic potential of rPso c 27 and rPsoSP2 by developing an iELISA. Compared to the rPsoSP2-iELISA, the rPso c 27-iELISA displayed better diagnostic efficiency, with higher values for sensitivity, specificity, and AUC (rPso c 27-rPsoSP2: 96.0-90.0, 90.91-78.18, and 0.988-0.964, respectively). Although rPso c 27 did show cross-reaction with sera from three of ten serum samples with S. scabiei infestations, the crossreaction between these two ectoparasites has been commonly shown in other studies [2, 29]. Fortunately, these two mite species were effectively treated with the same acaricide [3, 30]. Also, one in ten rabbits infested with S. scabiei, C. pisiformis, or Eimeria spp. showed a seroreaction with rPso c 27; however, their OD values were close to the cut-off value and appeared to markedly lower than those rabbits infested with $P$. ovis var. cuniculi $\left(F_{(1}\right.$ 
$\left.{ }_{78)}=115.444, P<0.0001\right)$. Moreover, the rPso c 27 -iELISA was able to detect seropositivity in $80 \%$ (8/10) of rabbits as early as week 2 p.i., prior to visible clinical signs and microscopy-positive skin scrapings. In terms of its high sensitivity and specificity, Pso c 27 can be considered to be the more suitable candidate antigen for serodiagnosis of $P$. ovis var. cuniculi infestation in rabbits, especially at the early stage of infestation.

\section{Conclusions}

In conclusion, Pso c 27 and PsoSP2 cDNAs displayed the typical characterization of the serpin superfamily, possessing the regular serpin domain and signature. The gene expression of Pso c 27 and PsoSP2 were found during all life stages of mites, with a significantly high expression in adult female mites. Compared to rPsoSP2, rPso c 27 seemed to display a better diagnostic efficiency than PsoSP2 by iELISA, suggesting that Pso c 27 could be developed as a potential antigen for serological diagnosis of $P$. ovis var. cuniculi infestation in rabbits, especially at the early stage of infestation.

\begin{abstract}
Abbreviations
AUC: Area under the receiver operating characteristic curve; Bf: Bootstrapping frequency; (i)ELISA: (Indirect) enzyme-linked immunosorbent assay; HRP: Horseradish peroxidase; NJ: Neighbor-joining; ORF: Open reading frame; p.i.: Post-infestation; RNAi: RNA interference; ROC: Receiver operating characteristic curve; rPso c 27/rPsoSP2: Recombinant Pso c 27/recombinant PsoSP2; serpin: Serine protease inhibitor.
\end{abstract}

\section{Acknowledgements}

We thank Youle Zheng, Xiaoxiao Yin, and Jiang Gu (Sichuan Agricultural University) for their help during serum collection and for their suggestions. We also thank Ruihui Zhang and Aftab Ahmed (Sichuan Agricultural University) for manuscript review and advice.

\section{Authors' contributions}

XBG conceived and designed the experiments. YHC and CYZ performed the experiments, inculding RT-PCR, qRT-PCR, recombinant protein expression, indirect ELISA. NXS and CW performed the tissue localization. XBG, YHC, YX, $\mathrm{RH}$, and $X Z$ performed the data analysis. GYY, XRP, DYY, and $Z H$ contributed reagents/materials/analysis tools. ZH and ZJZ provided experimental technical assistance. XBG and YHC wrote the initial manuscript. All authors read and approved the final manuscript.

\section{Funding}

This study received financial support from Sichuan Science \& Technology Program (Grant No. 2019YFN0155).

\section{Availability of data and materials}

The nucleotide sequences of serpin genes from $P$. ovis var. cuniculi in this article are available in the GenBank databases under the accession numbers MT707535 (Pso c 27) and MT707536 (PsoSP2). The other data supporting our findings and conclusions are available in the article.

\section{Ethics approval and consent to participate}

In this study, the animal procedure was reviewed and approved by the Animal Care and Use Committee of Sichuan Agricultural University (SYXK 2019-187). All the rabbits were strictly managed under the Guide for the Care and Use of Laboratory Animals (National Research Council, Bethesda, MD, USA) and the ARRIVE guidelines (https://www.nc3rs.org.uk/arrive-guidelines).
Consent for publication

Not applicable.

\section{Competing interests}

The authors declare that they have no competing interests.

\section{Author details}

${ }^{1}$ Department of Parasitology, College of Veterinary Medicine, Sichuan Agricultural University, Chengdu 611130, Sichuan, People's Republic of China. 2 Mianyang Animal Disease Control Center, Mianyang 621000, Sichuan, People's Republic of China. ${ }^{3}$ Institute of Animal Genetics and Breeding, College of Animal Science and Technology, Sichuan Agricultural University, Ya'an 625014, Sichuan, People's Republic of China. ${ }^{4}$ Department of Chemistry, College of Life and Basic Science, Sichuan Agricultural University, Ya'an 625014, Sichuan, People's Republic of China.

Received: 23 July 2020 Accepted: 24 November 2020

Published online: 11 December 2020

\section{References}

1. Siegfried $\mathrm{E}$, Ochs H, Deplazes P. Clinical development and serological. antibody responses in sheep and rabbits experimentally infested with Psoroptes ovis and Psoroptes cuniculi. Vet Parasitol. 2004;124:109-24.

2. Gu XB, Gu J, Ren YJ, Zheng YL, Yang GY, Zhou X, et al. Evaluation of an indirect ELISA using recombinant arginine kinase for serodiagnosis of Psoroptes ovis var. cuniculi infestation in rabbits. Front Vet Sci. 2019;6:411.

3. Elshahawy I, El-Goniemy A, Ali E. Epidemiological survey on mange mite of rabbits in the southern region of Egypt. Sains Malsys. 2016;45:745-51.

4. Sweatman GK. On the life history and validity of the species in Psoroptes, a genus of mange mites. Can J Zool. 1958;36:905-29.

5. Deloach JR, Wright FC. Ingestion of rabbit erythrocytes containing 51Cr-Labeled hemoglobin by Psoroptes spp. (Acari: Psoroptidae) that originated on cattle, mountain sheep, or rabbits. J Med Entomol. 1981;18:345-8.

6. Burgess STG, Frew D, Nunn F, Watkins CA, Mcneilly TN, Nisbet AJ, et al. Transcriptomic analysis of the temporal host response to skin infestation with the ectoparasitic mite Psoroptes ovis. BMC Genomics. 2010;11:624.

7. Broek AHVD, Huntley JF. Sheep Scab: the disease, pathogenesis and dontrol. J Comp Pathol. 2003;128:79-91.

8. Meekins DA, Kanost MR, Michel K. Serpins in arthropod biology. Semin Cell Dev Biol. 2017;62:105-19.

9. Bao J, Pan G, Poncz M, Wei J, Ran M, Zhou Z. Serpin functions in hostpathogen interactions. PeerJ. 2018;6:e4557.

10. Tanigawa C, Fujii Y, Miura M, Nzou SM, Mwangi AW, Nagi S, et al. Speciesspecific serological detection for Schistosomiasis by serine protease inhibitor (SERPIN) in multiplex assay. PLoS Neglect Trop D. 2015;9:e0004021.

11. Imamura S, Namangala B, Tajima T, Tembo ME, Yasuda J, Ohashi K, et al. Two serine protease inhibitors (serpins) that induce a bovine protective immune response against Rhipicephalus appendiculatus ticks. Vaccine. 2006;24:2230-7.

12. He ML, Xu J, He R, Shen NX, Gu XB, Peng XR, et al. Preliminary analysis of Psoroptes ovis transcriptome in different developmental stages. Parasites Vectors. 2016;9:570

13. Tamura K, Peterson D, Peterson N, Stecher G, Nei M, Kumar S. MEGA 5: Molecular Evolutionary Genetics Analysis using maximum likelihood, evolutionary distance, and maximum parsimony methods. Mol Biol Evol. 2011;28:2731-9.

14. Ochs H, Lonneux J, Losson BJ, Deplazes P. Diagnosis of psoroptic sheep scab with an improved enzyme-linked immunosorbent assay. Vet Parasitol. 2001;96:233-42.

15. Zheng Y, He R, He ML, Gu XB, Wang T, Lai WM, et al. Characterization of Sarcoptes scabiei cofilin gene and assessment of recombinant cofilin protein as an antigen in indirect-ELISA for diagnosis. BMC Infect Dis. 2016;16:21-8.

16. Crowther JR. The ELISA guidebook. New York: Humana Press; 2000.

17. Hanley JA, McNeil BJ. The meaning and use of the area under a receiver operating characteristic (ROC) curve. Radiology. 1982;143:29-36.

18. Casais R, Millán J, Rosell JM, Dalton KP, Prieto JM. Evaluation of an ELISA using recombinant $S s \lambda 20 \triangle B 3$ antigen for the serological diagnosis of 
Sarcoptes scabiei infestation in domestic and wild rabbits. Vet Parasitol. 2015;214:315-21.

19. Burgess STG, Marr EJ, Bartley K, Nunn FG, Down RE, Weaver RJ, et al. A genomic analysis and transcriptomic atlas of gene expression in Psoroptes ovis reveals feeding- and stage-specific patterns of allergen expression. BMC Genomics. 2019;20:756-78.

20. Gent DV, Sharp P, Morgan K, Kalsheker N. Serpins: structure, function and molecular evolution. Int J Biochem Cell B. 2003;35:1536-47.

21. Lin J, Li M, Liu Y, Jiang C, Wu Y, Wang Y, et al. Expression, purification and characterization of Der f 27, a new allergen from Dermatophagoides farinae. Am J Transl Res. 2015;7:1260-70

22. Burgess STG, Mcneilly TN, Watkins CA, Nisbet AJ, Huntley JF. Host transcription factors in the immediate pro-inflammatory response to the parasitic mite Psoroptes ovis. PLoS ONE. 2011;6:e24402.

23. Pascini TV, Martins GF. The insect spermatheca: an overview. Zoology. 2017:121:56-71.

24. Xu Z, Yan Y, Zhang H, Cao J, Zhou Y, Xu Q, et al. A serpin from the tick Rhipicephalus haemaphysaloides: involvement in vitellogenesis. Vet Parasitol. 2020;279:109064.

25. Swe PM, Katja F. A scabies mite serpin interferes with complement-mediated neutrophil functions and promotes staphylococcal growth. PLoS Neglect Trop Dis. 2014;8(6):e2928.
26. Mika A, Reynolds SL, Mohlin FC, Willis C, Swe PM, Pickering DA, et al. Novel scabies mite serpins inhibit the three pathways of the human complement system. PLOS ONE. 2012;7:e40489.

27. Bo KR. Investigation on psoroptic mange of in Gangou township, Minhe county. Chinese Qinghai J Animal Vet Sci. 2012;3:14.

28. Uhliř J. Humoral and cellular immune response of rabbits to Psoroptes cuniculi, the rabbit scab mite. Vet Parasitol. 1991;40:325-34.

29. Zheng WP, Zhang RH, Wu XH, Ren YJ, Nong X, Gu XB, et al. Evaluating troponin $C$ from Psoroptes cuniculi as a diagnostic antigen for a dotELISA assay to diagnose mite infestations in rabbits. Parasite Immunol. 2014;36(2):53-9.

30. Divisha R, Soundararajan C, Prakash MA. Therapeutic management of concurrent sarcoptic and psoroptic mange infestation in rabbits. J Entomol Zool Stud. 2020:8:1041-3.

\section{Publisher's Note}

Springer Nature remains neutral with regard to jurisdictional claims in published maps and institutional affiliations.
Ready to submit your research? Choose BMC and benefit from:

- fast, convenient online submission

- thorough peer review by experienced researchers in your field

- rapid publication on acceptance

- support for research data, including large and complex data types

- gold Open Access which fosters wider collaboration and increased citations

- maximum visibility for your research: over $100 \mathrm{M}$ website views per year

At BMC, research is always in progress.

Learn more biomedcentral.com/submissions 